\title{
Von «Wirtschaftlichkeitskontrollen», der Abschaffung des Kontrahierungszwangs und dem drohenden Begräbnis unseres psychosozialen Gesundheitswesens
}

\author{
P. Schindler
}

Korrespondenz:

Dr. med. Peter Schindler Facharzt FMH für Psychiatrie und Psychotherapie Eulerstr. 17

CH-Basel
Im September erhielten zahlreiche Psychiater/ innen in Basel und Baselland einen Brief der Santésuisse mit unglaublich massiven Drohungen. Es stand darin im letzten Abschnitt: «Gemäss unseren jetzigen Erkenntnissen müssen wir davon ausgehen, dass Sie das Gebot der Wirtschaftlichkeit gemäss Artikel 56 des KVG nicht einhalten. Wir teilen Ihnen daher mit, dass wir uns für das Behandlungsjahr 2001 Rückforderungen vorbehalten müssen, wenn sich Ihr Index Arztkosten gemäss Statistikblatt 2002 nicht klar verbessert.» Dies wohlverstanden 3 Monate vor Jahresende 2002. Kein Wunder, dass viele Kollegen/-innen sich massiv bedroht fühlen, haben sie doch nicht anders als seit Jahren ihren Beruf nach bestem Wissen und Gewissen und lege artis nach den notwendigen Regeln der ärztlichen psychiatrisch-psychotherapeutischen Wissenschaft ausgeführt und wissen gar nicht, wie sie ihren Index Arztkosten «klar verbessern» sollen (zudem gehören die Psychiater/innen mit ihrem möglichen Einkommen an der untersten Grenze aller Ärzteeinkommen ja wirklich nicht zu den «Abzockern» im Gesundheitswesen. Wer da noch Rückzahlungen leisten müsste, wäre ganz klar existentiell bedroht).

Was geschieht da eigentlich? Die Santésuisse legt einfach einen Sollwert der Wirtschaftlichkeit, den sogenannten ominösen Fallkostenindex, von 100 fest, was etwa 2000 Franken Behandlungskosten pro Fall und Jahr bedeutet. Da die Psychiater/innen im Zeittarif arbeiten, heisst das, ein Fall darf maximal 12 Psychotherapiestunden pro Jahr, also eine Stunde pro Monat, beanspruchen, sonst wird er unwirtschaftlich.

Das ist natürlich absurd und nicht Wirtschaftlichkeitskontrolle, sondern statistischer Unsinn. Ernsthafte und effektive, aufdeckende Psychotherapie, die medizinisch überhaupt verantwortbar und nicht ein Kunstfehler ist, muss normalerweise mit einer oder sogar zwei Wochenstunden durchgeführt werden, deshalb ist das im Gesetz auch so verankert (gemäss der
Krankenpflege-Leistungsverordnung KLV, Art. 2, erlassen vom EDI 1995, müssen die Kassen, sofern es medizinisch indiziert und von den Vertrauensärzten bestätigt wird, drei Jahre lang zwei Stunden und weitere drei Jahre eine Stunde Psychotherapie pro Woche bezahlen, danach eine einstündige Sitzung alle zwei Wochen). Dies hat auch das Eidgenössische Versicherungsgericht in mehreren Urteilen bestätigt. Rückforderungen der Versicherer wegen zeitaufwendiger psychiatrischer Behandlungen wurden bisher jedes Mal abgewiesen. Das scheint die Santésuisse aber nicht im mindesten zu beeindrucken.

Auch Kriseninterventionen, die sehr oft teure und traumatische Hospitalisationen und andere dramatische Entwicklungen verhindern können, erfordern oft zeitintensive Therapien, ebenso komplexe sozialpsychiatrische Aufgaben, schwierige Familientherapien und anderes mehr.

Wer diese Aufgaben lege artis und kompetent gemäss seiner Ausbildung, den wissenschaftlich anerkannten Kriterien und seinem ärztlichen Gewissen ausführt, wird jetzt von der Santésuisse massiv bedroht und - das liegt auf der Hand - bei Aufhebung des Kontrahierungszwanges von den Verträgen ausgeschlossen.

Das heisst im Klartext, dass das Gesetz (KLV) zur Makulatur wird und verantwortliche, effektive und qualitativ genügende psychiatrisch-psychotherapeutische Versorgung unserer Patienten nicht mehr möglich sein wird.

Es wird $\mathrm{zu}$ einer massiven Zunahme der Hospitalisationen in den jetzt schon überfüllten Kliniken kommen, zu einer gravierenden Verschlechterung der Prävention, Betreuung und Heilungsmöglichkeiten von psychischen Störungen, welche ohnehin aufgrund der aktuellen komplexen gesellschaftlichen Entwicklungen zunehmen und uns vor immer schwerwiegendere Probleme stellen.

Die WHO berichtet von einer allgemeinen Tendenz zur Schlechterstellung von psychisch Kranken. 50\% der Obdachlosen leiden zum Beispiel gemäss einer vor fünf Jahren gemachten 
englischen Studie an ernsthaften, psychiatrisch behandlungsbedürftigen Erkrankungen. Alltägliche Gewalt erschüttert immer mehr unsere Welt. Wir sind geschockt über das Ausmass von Kindsmissbrauch und Pädophilie, welche in der aktuellen Internet-Pornoaffäre aufgedeckt wird. Wir erhalten Notrufe von Behörden zur Lösung der psychiatrisch-psychotherapeutischen Unterversorgung von traumatisierten Menschen, Folteropfern, gewaltbetroffenen Kindern. Auch in Basel gibt es beispielsweise schon Schulen, wo der Abwart eine Waffe mit sich trägt. Mehr als doppelt so viele Menschen sterben in unserem Land an Selbstmord wie an Verkehrsunfällen. Das Thema psychische Gesundheit ist nicht umsonst Schwerpunkt der «Arbeitstagung nationale Gesundheitspolitik» von Bund und Kantonen in diesem Herbst.

Wir brauchen in Zukunft eine Verbesserung der psychiatrisch-psychotherapeutischen Versorgung und nicht einen Abbau. In einem arbeitsintensiven Prozess haben wir in der Schweiz in den letzten Jahren und Jahrzehnten auf der Basis der sozialen Krankenversicherung ein gut funktionierendes und sehr ausgelastetes psychiatrisch-psychotherapeutisches Versorgungsnetz mit hochqualifizierten Psychiatern/-innen und Psychotherapeuten/-innen für alle aufgebaut. Dies trägt zu einem grossen Teil zu unser aller Lebensqualität bei und kostet natürlich etwas. Wollen wir dieses komplexe und effiziente
Versorgungsnetz nun von unterinformierten Politikern/-innen und Krankenkassenverantwortlichen zerstören lassen? Wenn ich in der SÄZ vom 31.10.2002 (S. 2337) dann noch lese, dass unser FMH-Präsident in einem Interview gesagt hat, die psychosoziale Medizin werde wohl aus dem Pflichtleistungskatalog der Krankenversicherung verschwinden, läuft es mir kalt den Rücken hinunter ...

Nein, ein engagiertes Auftreten und Kämpfen für eine qualitativ hochstehende Versorgung aller psychisch leidenden Menschen in unserer Gesellschaft ist jetzt unsere Pflicht. Ich möchte an alle Kolleginnen und Kollegen appellieren, aufzuwachen, bevor es zu spät ist. Wir können es uns nicht mehr leisten, in vornehmer Zurückhaltung abzuwarten, was passiert. Es ist mindestens eine Minute vor Zwölf. Wir müssen als die Fachexperten/-innen mit der besten und längsten Ausbildung und mit dem grössten Wissen in psychosomatischen und psychosozialen Belangen unsere gesundheitspolitische Verantwortung wahrnehmen, uns einmischen, an die Öffentlichkeit gehen, mit den Politikern/-innen reden und last but not least die Vertreter/-innen an der Spitze unserer Standesorganisation FMH überzeugen, sich auch für die Sicherung der psychiatrisch-psychotherapeutischen Versorgung unserer Bevölkerung engagiert einzusetzen. 